\title{
CRLB-based Positioning Performance of Indoor Hybrid AoA/RSS/ToF Localization
}

\author{
Chenglong Li ${ }^{1 \dagger}$, Jens Trogh ${ }^{1}$, David Plets ${ }^{1}$, Emmeric Tanghe ${ }^{1}$, Jeroen Hoebeke ${ }^{2}$, \\ Eli De Poorter ${ }^{2}$, Wout Joseph ${ }^{1}$ \\ ${ }^{1}$ INTEC-WAVES, Ghent University-IMEC, Ghent, Belgium \\ 2 INTEC-IDLab, Ghent University-IMEC, Ghent, Belgium \\ $\dagger$ Email: chenglong.li@ugent.be
}

\begin{abstract}
Fingerprinting indoor localization provides high positioning accuracy with low cost and easy deployment. Considering the unsatisfying precision of received signal strength (RSS)based fingerprinting, hybrid metrics including angle-of-arrival (AoA) and time-of-flight (ToF), are incorporated to the RSS fingerprinting system. To evaluate the positioning performance of hybrid metrics, the closed-form Cramér-Rao lower bound (CRLB) is derived in this paper. The existence conditions of CRLBs, as well as the relationship of the CRLBs between single and hybrid metrics is revealed. Numerical results based on an office building scenario show that hybrid metrics greatly improve the positioning performance and the robustness to measured standard deviations compared to the single metric's case. Furthermore, hybrid schemes of the AoA/RSS/ToF metrics are also investigated, and simulations reveal that the scheme of AoA/ToF-supporting access points (AP) enhanced with single RSS-supporting APs achieves the best positioning accuracy among all hybrid schemes.
\end{abstract}

\section{INTRODUCTION}

Indoor localization has attracted a growing interest recently due to the easy deployment of low-cost infrastructure, such as wireless local area networks, ultra-wideband (UWB), wireless sensor networks, and radio frequency identification [1]. Due to the limitation of global navigation satellite system's signal strength for indoor scenarios, nearby access points (AP) with known positions are generally needed for an indoor positioning system. A variety of positioning algorithms have been developed, mainly including range-based and range-free schemes [2], [3], while range-based approaches are favored for accurate positioning.

Location fingerprinting techniques are quite promising for fine-grain indoor localization [4], where location-dependent parameters are collected as location fingerprints in an off-line phase. During the online positioning phase, the parameters obtained are compared with the database to estimate the mobile nodes' location. Currently, due to the relatively low deployment cost and the compatibility with WiFi- or Bluetooth Low Energy (BLE)-enabled off-the-shelf devices [5], the received signal strength (RSS)-based fingerprinting approach becomes a popular solution. However, RSS-based positioning does not always provide reliable location information as a result of complex indoor propagation loss and channel fading. The positioning performance will greatly improve if angleof-arrival (AoA) and time-of-flight (ToF) are incorporated in the RSS-based fingerprinting framework [5], which provides multiple metrics to improve localization accuracy. In [6], based on Decawave's DW1000 impulse radio UWB IC, the Decawave's AoA estimation demo was described. Together with the ToF estimation scheme in [7], such kind of hardware would pave the way for hybrid time-based and AoA-based indoor localization with the aim of a finer-grain positioning performance.

Cramér-Rao lower bound (CRLB) has been widely used as a benchmark as it gives the performance limit of unbiased estimator [8]. As an efficient tool to evaluate the positioning accuracy, the CRLBs of single metric, such as AoA, RSS and ToF, have been derived in [9]-[11]. In [12], the CRLBs of hybrid time (difference) of arrival (ToA/TDoA) and RSS were derived for wireless sensing network, and the CRLBs of hybrid ToA/AoA for UWB-based scheme and passive cooperative localization in [13], [14], respectively. In this paper, we derive the closed-form CRLB for hybrid AoA/RSS/ToF fingerprinting systems. According to the authors' best knowledge, it is the first time to derive the CRLB for hybrid metrics assuming that not each AP measures all AoA/RSS/ToF metrics simultaneously, which provides flexible deployment for practical applications.

The reminder of this paper is organized as follows. Section II presents the localization model with hybrid metrics. The CRLB of hybrid metrics is derived in Section III, and some useful conclusions are presented and proved. In Section IV, the positioning performance of hybrid metrics is analyzed under an indoor office scenario. Section V concludes this paper.

\section{HYBRID AOA/RSS/ToF LOCALIZATION}

Consider a hybrid indoor localization scheme with $N$ APs, where the coordinates of the APs are defined as $\mathbf{p}_{i}=$ $\left[x_{i}, y_{i}\right]^{\top} \in \mathbb{R}^{2}, i \in\{1,2, \cdots, N\}$, and mobile node is denoted as $\mathbf{p}=[x, y]^{\top} \in \mathbb{R}^{2}$, as shown in Fig. 1. We assume that each AP can perform at least one of the AoA, RSS and ToF measurements. The AoA $\alpha_{i}$ is defined as

$$
\alpha_{i}=\arctan \frac{y_{i}-y}{x_{i}-x},
$$

where $\alpha_{i} \in[-\pi / 2, \pi / 2]$ when considering the threshold conditions at $\pm \pi / 2$. As shown in Fig. 1, the positive AoA is the counter-clockwise angle rotation from right-hand axis when AP facing with mobile node, while the negative AoA 


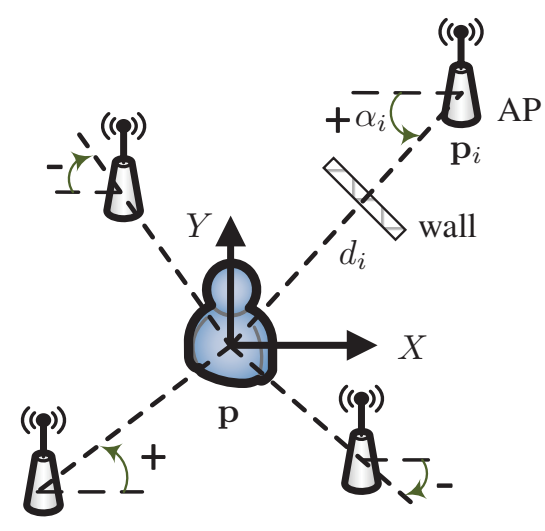

Fig. 1. Distribution of APs and mobile node.

is the clockwise rotation. The AoA measurement can be expressed as $\hat{\alpha}_{i}=\alpha_{i}+\varpi$, where $\varpi$ is the measured error following the Gaussian distribution [9], [13], [14] with zero mean and variance $\sigma_{\alpha i}^{2}$. According to [15], the total indoor path loss for a path between the transmitter and the receiver location, is the sum of the the distance loss along the path, the total wall loss along the path, and the interaction loss along the path. The total path loss can be calculated as follows [15]

$$
P_{i}=P\left(d_{0}\right)-10 \beta \log _{10}\left(d_{i} / d_{0}\right)+\sum L_{W}+\sum L_{I}+\chi,
$$

where $P_{i}$ and $P\left(d_{0}\right)$ are the received power at AP and reference distance $d_{0}$. $\beta$ represents the path loss exponent, $d_{i}=\left\|\mathbf{p}_{i}-\mathbf{p}\right\|_{2}$ is the distance between AP and mobile node. The first two terms of the sum represent the path loss due to the distance along the considered path. $\sum L_{W}$ is the cumulated wall loss along the path, $\sum L_{I}$ represents the cumulated interaction loss due to the propagation direction changing from mobile node to AP. $\chi$ denotes the Log-normal shadowing term following the Gaussian distribution as $\chi \sim \mathcal{N}\left(0, \sigma_{P i}^{2}\right)$. The measurement of $\mathrm{ToF}$ is the time from mobile node to AP $\tau_{i}=d_{i} / c$, where $c$ is the speed of electromagnetic wave. The measured error of ToF is also assumed to follow a Gaussian distribution [11]-[14] with variance $\sigma_{\tau i}^{2}$.

In the hybrid AoA/RSS/ToF localization, the APs obtain at least one of these three metrics in practical applications. We assume that $m$ of the $N$ APs can perform AoA measurements, $n$ of $N$ APs perform RSS measurements, $l$ of $N$ APs perform ToF measurements, and the observations are independent of each other. So the hybrid metrics can be redefined as $\mathfrak{M}=$ $\left\{\alpha_{1}, \cdots, \alpha_{m}, P_{1}, \cdots, P_{n}, \tau_{1}, \cdots, \tau_{l}\right\},\{m, n, l\} \in[1, N]$. The joint probability density function (PDF) of hybrid metrics is given as

$$
f(\hat{\boldsymbol{\theta}} \mid \mathbf{p})=\prod_{i=1}^{m} f\left(\alpha_{i} \mid \mathbf{p}\right) \prod_{j=1}^{n} f\left(P_{j} \mid \mathbf{p}\right) \prod_{k=1}^{l} f\left(\tau_{k} \mid \mathbf{p}\right),
$$

where $\hat{\boldsymbol{\theta}}$ is given by $\hat{\boldsymbol{\theta}}=\left(\hat{\boldsymbol{\alpha}}^{\top}, \hat{\mathbf{P}}^{\top}, \hat{\boldsymbol{\tau}}^{\top}\right)^{\top}, \hat{\boldsymbol{\alpha}}$ is the estimation of AoA as $\hat{\boldsymbol{\alpha}}=\left(\hat{\alpha}_{1}, \hat{\alpha}_{2}, \cdots, \hat{\alpha}_{m}\right)^{\top}, \hat{\mathbf{P}}$ the estimation of RSS as $\hat{\mathbf{P}}=\left(\hat{P}_{1}, \hat{P}_{2}, \cdots, \hat{P}_{n}\right)^{\top}$, and $\hat{\boldsymbol{\tau}}$ the estimation of ToF as $\hat{\tau}=\left(\hat{\tau}_{1}, \hat{\tau}_{2}, \cdots, \hat{\tau}_{l}\right)^{\top}$. Based on the maximum likelihood estimation, the position of the mobile node can be determined by maximizing the Log-likelihood function, namely

$$
\mathbf{p}=\underset{\mathbf{p}}{\arg \max } \ln f(\hat{\boldsymbol{\theta}} \mid \mathbf{p}),
$$

where $\ln f(\hat{\boldsymbol{\theta}} \mid \mathbf{p})$ is further given by

$$
\begin{aligned}
\ln f(\hat{\boldsymbol{\theta}} \mid \mathbf{p}) & =\sum_{i=1}^{m} \ln f\left(\alpha_{i} \mid \mathbf{p}\right)+\sum_{i=1}^{n} \ln f\left(P_{i} \mid \mathbf{p}\right)+\sum_{i=1}^{l} \ln f\left(\tau_{i} \mid \mathbf{p}\right) \\
& =\mathcal{C}-\frac{1}{2}\left[(\hat{\boldsymbol{\theta}}-\boldsymbol{\theta}(\mathbf{p}))^{\top} \mathbf{R}^{-1}(\hat{\boldsymbol{\theta}}-\boldsymbol{\theta}(\mathbf{p}))\right],
\end{aligned}
$$

where $\mathcal{C}$ is denoted as

$\mathcal{C}=\sum_{i=1}^{m} \ln \left(\frac{1}{\sqrt{2 \pi} \sigma_{\alpha_{i}}}\right)+\sum_{i=1}^{n} \ln \left(\frac{1}{\sqrt{2 \pi} \sigma_{P_{i}}}\right)+\sum_{i=1}^{l} \ln \left(\frac{1}{\sqrt{2 \pi} \sigma_{\tau_{i}}}\right)$,

and $\boldsymbol{\theta}(\mathbf{p})$ is given by $\boldsymbol{\theta}(\mathbf{p})=\left(\boldsymbol{\alpha}^{\top}(\mathbf{p}), \mathbf{P}^{\top}(\mathbf{p}), \boldsymbol{\tau}^{\top}(\mathbf{p})\right)^{\top}$, where $\boldsymbol{\alpha}(\mathbf{p})=\left(\alpha_{1}(\mathbf{p}), \alpha_{2}(\mathbf{p}), \cdots, \alpha_{m}(\mathbf{p})\right)^{\top}$ represent the true AoAs with mobile node's coordinates from $m$ APs, $\mathbf{P}(\mathbf{p})=\left(P_{1}(\mathbf{p}), P_{2}(\mathbf{p}), \cdots, P_{n}(\mathbf{p})\right)^{\top}$ denotes the true RSS from $n$ APs, and $\boldsymbol{\tau}(\mathbf{p})=\left(\tau_{1}(\mathbf{p}), \tau_{2}(\mathbf{p}), \cdots, \tau_{l}(\mathbf{p})\right)^{\top}$ is the ToF from $l$ APs. $\mathbf{R}=\operatorname{diag}\left(\mathbf{R}_{\alpha}, \mathbf{R}_{P}, \mathbf{R}_{\tau}\right)$, where $\mathbf{R}_{\alpha}, \mathbf{R}_{P}$, and $\mathbf{R}_{\tau}$ are the error covariance matrixes of AoA, RSS, and ToF, respectively.

\section{CRLB OF HYBRID METRICS}

For an unbiased estimator, the estimation variance is bounded by the CRLB, which is the inverse of the Fisher Information Matrix (FIM) $\mathbf{I}(\mathbf{p})$. The CRLB of hybrid AoA/RSS/ToF localization for mobile node is defined as the summation of the CRLB of each coordinate

$$
\sigma_{C}^{2}(\mathbf{p})=\sigma_{C}^{2}(x)+\sigma_{C}^{2}(y)=\operatorname{tr}\left(\mathbf{I}^{-1}(\mathbf{p})\right) .
$$

Notice that $\mathbf{I}$ is nonsingular, the proof will be given later. The FIM is defined as

$$
\begin{aligned}
\mathbf{I}(\mathbf{p}) & =\mathbb{E}\left(\nabla \ln f(\hat{\boldsymbol{\theta}} \mid \mathbf{p}) \nabla \ln f(\hat{\boldsymbol{\theta}} \mid \mathbf{p})^{\top}\right) \\
& =-\mathbb{E}\left(\nabla^{2} \ln f(\hat{\boldsymbol{\theta}} \mid \mathbf{p})\right)=-\mathbb{E}(\mathbf{H}(\mathbf{p})),
\end{aligned}
$$

where $\nabla, \nabla^{2}$ are the operator of the first and second order differentiation, respectively, which are with respect to mobile node's position $\mathbf{p}$ in this paper. $\mathbf{H}(\mathbf{p})$ is the Hessian matrix. According to (5), we have

$$
\nabla \ln f(\hat{\boldsymbol{\theta}} \mid \mathbf{p})=\nabla \boldsymbol{\theta}^{\top}(\mathbf{p}) \mathbf{R}^{-1}(\hat{\boldsymbol{\theta}}-\boldsymbol{\theta}(\mathbf{p})) .
$$

Substitute (8) into (7), we obtain

$$
\mathbf{I}(\mathbf{p})=\nabla \boldsymbol{\theta}^{\top}(\mathbf{p}) \mathbf{R}^{-1} \nabla \boldsymbol{\theta}(\mathbf{p}) .
$$

Based on the definitions of $\boldsymbol{\theta}$ and $\mathbf{R}$, the following relationship between the FIM of hybrid metrics and single metrics' FIMs can be given by $\mathbf{I}(\mathbf{p})=\mathbf{I}_{\alpha}(\mathbf{p})+\mathbf{I}_{P}(\mathbf{p})+\mathbf{I}_{\tau}(\mathbf{p})$. Here, $\mathbf{I}_{\Xi}(\mathbf{p})$ $(\Xi \in\{\alpha, P, \tau\})$ is given as

$$
\begin{aligned}
\mathbf{I}_{\Xi}(\mathbf{p}) & =\nabla \Xi^{\top}(\mathbf{p}) \mathbf{R}_{\Xi}^{-1} \nabla \boldsymbol{\Xi}(\mathbf{p}) \\
& =\sum_{i=1}^{\nu} \frac{1}{\sigma_{\Xi i}^{2}} \nabla \Xi_{i}(\mathbf{p})\left(\nabla \Xi_{i}(\mathbf{p})\right)^{\top},
\end{aligned}
$$


where $\nu \in\{m, n, l\}$. So the CRLB of hybrid metrics is obtained as below

$$
\begin{aligned}
\sigma_{C}^{2}(\mathbf{p}) & =\frac{\operatorname{tr}(\mathbf{I}(\mathbf{p}))}{\operatorname{det}(\mathbf{I}(\mathbf{p}))}=\frac{\operatorname{tr}\left(\mathbf{I}_{\alpha}(\mathbf{p})+\mathbf{I}_{\mathrm{P}}(\mathbf{p})+\mathbf{I}_{\tau}(\mathbf{p})\right)}{\operatorname{det}(\mathbf{I}(\mathbf{p}))} \\
& =\kappa_{\alpha} \sigma_{C \alpha}^{2}(\mathbf{p})+\kappa_{P} \sigma_{C P}^{2}(\mathbf{p})+\kappa_{\tau} \sigma_{C \tau}^{2}(\mathbf{p}),
\end{aligned}
$$

where $\kappa_{\Xi}=\operatorname{det}\left(\mathbf{I}_{\Xi}(\mathbf{p})\right) / \operatorname{det}(\mathbf{I}(\mathbf{p})), \Xi \in\{\alpha, P, \tau\}$, which reveals that the CRLB of hybrid metrics is the linear summation of CRLB of single metric $\sigma_{C \Xi}^{2}(\mathbf{p})=\operatorname{tr}\left(\mathbf{I}_{\Xi}^{-1}(\mathbf{p})\right)$, where $\mathbf{I}_{\Xi}(\mathbf{p})$ should be non-singular.

Remark 1. Single Metric: To guarantee the existence of single metric's CRLB, the APs and mobile node should not be placed collinearly.

Proof. According to the geometry relationship between AoA and the coordinates, as shown in Fig. 1, the FIMs of AoA, $\mathrm{RSS}$, and ToF metric can be expressed as

$$
\begin{aligned}
& \mathbf{I}_{\alpha}(\mathbf{p})=\sum_{i=1}^{m} \gamma_{\alpha i}^{2} \mathbf{M}_{i}, \\
& \mathbf{I}_{P}(\mathbf{p})=\sum_{i=1}^{n} \gamma_{P i}^{2} \mathbf{M}_{i}^{*}, \\
& \mathbf{I}_{\tau}(\mathbf{p})=\sum_{i=1}^{l} \gamma_{\tau i}^{2} \mathbf{M}_{i}^{*},
\end{aligned}
$$

where

$$
\begin{gathered}
\gamma_{\alpha i}^{2}=\frac{1}{d_{i}^{2} \sigma_{\alpha i}^{2}}, \gamma_{P i}^{2}=\left(\frac{10 \beta}{\ln 10}\right)^{2} \frac{1}{d_{i}^{2} \sigma_{P i}^{2}}, \gamma_{\tau i}^{2}=\frac{1}{c^{2} \sigma_{\tau i}^{2}}, \\
\mathbf{M}_{i}=\left(\begin{array}{cc}
\sin ^{2} \alpha_{i} & -\sin \alpha_{i} \cos \alpha_{i} \\
-\sin \alpha_{i} \cos \alpha_{i} & \cos ^{2} \alpha_{i}
\end{array}\right),
\end{gathered}
$$

and $\mathbf{M}_{i}^{*}$ is the adjoint matrix of $\mathbf{M}_{i}$. Thus, the determinant of the FIM is given as

$$
\operatorname{det}\left(\mathbf{I}_{\Xi}(\mathbf{p})\right)=\sum_{i=1}^{\nu} \sum_{j=1}^{\nu} \frac{\cos \alpha_{i} \sin \alpha_{j} \sin \left(\alpha_{j}-\alpha_{i}\right)}{\left(1 / \gamma_{\Xi i}^{2}\right)\left(1 / \gamma_{\Xi j}^{2}\right)} .
$$

Specifically, to satisfy that the CRLB of AoA metric exists, the FIM should be non-singular, namely $\operatorname{det}\left(\mathbf{I}_{\alpha}(\mathbf{p})\right) \neq 0$. When set $\operatorname{det}\left(\mathbf{I}_{\alpha}(\mathbf{p})\right)=0$, we have $\alpha_{i}=\pi / 2$ for any $i \in\{1,2, \cdots, m\}$, or $\alpha_{j}=0$ for any $j \in\{1,2, \cdots, m\}$, or $\alpha_{i}=\alpha_{j} \pm\{0, \pi\}$ for any $i, j \in\{1,2, \cdots, m\}$. To sum up three cases, the APs and mobile node should not be placed collinearly to guarantee the existence of CRLB of AoA metric. For the other two metrics, the same conclusion is reached.

Remark 2. Hybrid Metrics: To guarantee the existence of RSS/ToF's CRLB, the APs and mobile node should not be placed collinearly. For hybrid AoA/RSS, AoA/ToF, and AoA/RSS/ToF localization, the CRLBs always exist.

Proof. For hybrid RSS/ToF metrics, the FIM is given as follows

$$
\mathbf{I}_{P \tau}(\mathbf{p})=\mathbf{I}_{P}(\mathbf{p})+\mathbf{I}_{\tau}(\mathbf{p})=\sum_{i=1}^{n} \gamma_{P i}^{2} \mathbf{M}_{i}^{*}+\sum_{i=1}^{l} \gamma_{\tau i}^{2} \mathbf{M}_{i}^{*}
$$

Without loss of generality, we assume that $n>l$, and set $\tilde{\gamma}_{\tau}^{2}=$ $\left[\gamma_{\tau 1}^{2}, \gamma_{\tau 2}^{2}, \cdots, \gamma_{\tau l}^{2}, 0, \cdots, 0\right]_{1 \times n}=\left\{\tilde{\gamma}_{\tau i}^{2} \mid i=1,2, \cdots, n\right\}$. So (16) can be modified as

$$
\mathbf{I}_{P \tau}(\mathbf{p})=\sum_{i=1}^{n}\left(\gamma_{P i}^{2}+\tilde{\gamma}_{\tau i}^{2}\right) \mathbf{M}_{i}^{*}=\sum_{i=1}^{n} \gamma_{i}^{2} \mathbf{M}_{i}^{*},
$$

where $\gamma_{i}^{2}=\gamma_{P i}^{2}+\tilde{\gamma}_{\tau i}^{2}$, thus (17) has the same form as (13). Similar to the single RSS metric, we can draw the conclusion that the RSS/ToF's CRLB exists when the APs and mobile node are not placed collinearly.

For hybrid AoA/RSS metrics, we have the FIM as follows

$$
\mathbf{I}_{\alpha P}(\mathbf{p})=\sum_{i=1}^{m} \gamma_{\alpha i}^{2} \mathbf{M}_{i}+\sum_{i=1}^{n} \gamma_{P i}^{2} \mathbf{M}_{i}^{*}=\sum_{i=1}^{m} \boldsymbol{\Omega}_{i},
$$

in which we assume that $m>n$, and expand the coefficient vector as $\tilde{\gamma}_{P}^{2}=\left[\gamma_{P 1}^{2}, \gamma_{P 2}^{2}, \cdots, \gamma_{P n}^{2}, 0, \cdots, 0\right]_{1 \times m}=$ $\left\{\tilde{\gamma}_{P i}^{2} \mid i=1,2, \cdots, m\right\}$. Here,

$\boldsymbol{\Omega}_{i}=\left(\begin{array}{cc}\gamma_{\alpha i}^{2} \sin ^{2} \alpha_{i}+\tilde{\gamma}_{P i}^{2} \cos ^{2} \alpha_{i} & \left(\tilde{\gamma}_{P i}^{2}-\gamma_{\alpha i}^{2}\right) \sin \alpha_{i} \cos \alpha_{i} \\ \left(\tilde{\gamma}_{P i}^{2}-\gamma_{\alpha i}^{2}\right) \sin \alpha_{i} \cos \alpha_{i} & \gamma_{\alpha i}^{2} \cos ^{2} \alpha_{i}+\tilde{\gamma}_{P i}^{2} \sin ^{2} \alpha_{i}\end{array}\right)$

is positive definite, which can be easily proved. So $\mathbf{I}_{\alpha P}(\mathbf{p})$ is the positive definite and non-singular. Likewise, it can be concluded that the FIMs of AoA/ToF and AoA/RSS/ToF are also non-singular, namely the CRLBs always exist.

Remark 3. The CRLB of hybrid AoA/RSS/ToF metrics is less than any of single metric's CRLB, also less than the CRLB of any two hybrid metrics.

Proof. Since $\operatorname{tr}(\mathbf{I}(\mathbf{p}))=\operatorname{tr}\left(\sum_{\Xi} \mathbf{I}_{\Xi}(\mathbf{p})\right)>\operatorname{tr}\left(\mathbf{I}_{\Xi}(\mathbf{p})\right), \Xi \in$ $\{\alpha, P, \tau\}$, we obtain $\sigma_{C}^{2}(\mathbf{p})=\operatorname{tr}\left(\mathbf{I}^{-1}(\mathbf{p})\right)<\operatorname{tr}\left(\mathbf{I}_{\Xi}^{-1}(\mathbf{p})\right)=$ $\sigma_{C \Xi}^{2}(\mathbf{p})$, when the FIM of single metric is positive definite according to Remark 1. It reveals that the CRLB of hybrid AoA/RSS/ToF metrics is less than any of single metric's CRLB. Likewise, the CRLB of hybrid AoA/RSS/ToF metrics is also less than the CRLB of any two hybrid metrics.

\section{Simulations And Results}

\section{A. Simulation Configuration}

To evaluate the CRLB of hybrid metrics localization, an indoor scenario of iGent office building floor as shown in Fig. 2, measuring $27 \mathrm{~m} \times 41 \mathrm{~m}$, is utilized. The floor plan data of the building is generated by the WHIPP propagation prediction software [15], [16]. In the simulations, different configurations with a varying number of APs (from 5 to 39) will be considered, when each AP is assumed to measure at least one of AoA/RSS/ToF. In Fig. 2, the blue filled circles are the APs, and the segments with different color represent different types of material (black: concrete, red: metal, grey: glass, yellow: wood, and green: layered drywall). For each configuration, we assume that the mobile nodes are deployed on a rectangular grid with a spacing of 2.6 meters.

The observation standard deviation of AoA is related with specific indoor environment [5], [17]. In the simulations, $\sigma_{\alpha i}$ $(i \in 1,2, \cdots, m)$ are set to be from 6 to 15 in degree. As for 


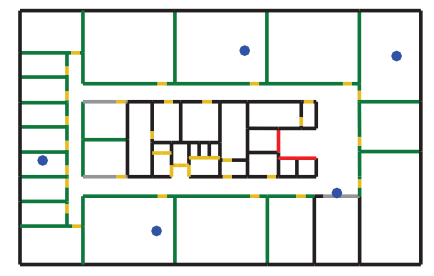

(a) $5 \mathrm{APs}$

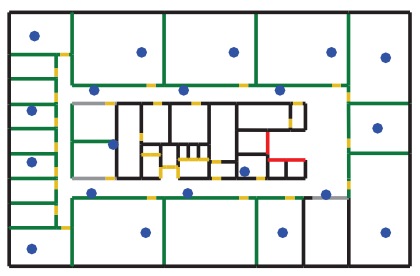

(e) $20 \mathrm{APs}$

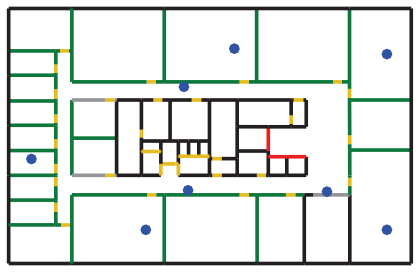

(b) $8 \mathrm{APs}$

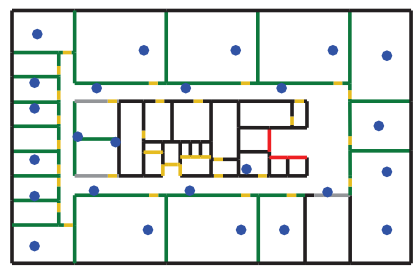

(f) $25 \mathrm{APs}$

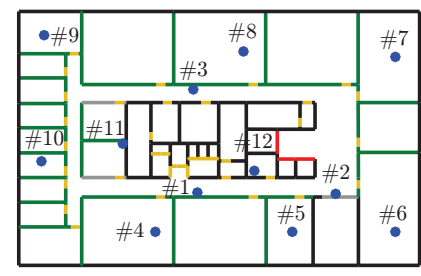

(c) $12 \mathrm{APs}$

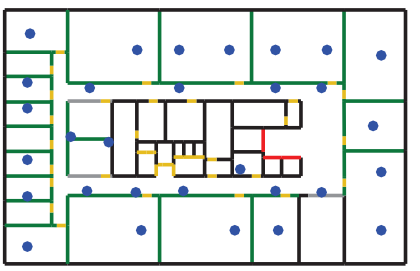

(g) $30 \mathrm{APs}$

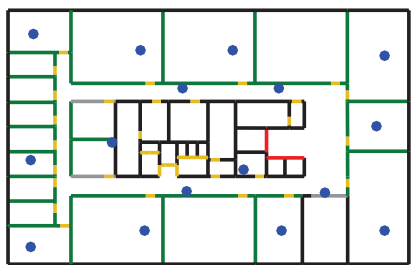

(d) $16 \mathrm{APs}$

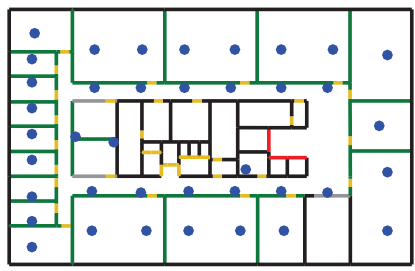

(h) $39 \mathrm{APs}$

Fig. 2. The floor plan of iGent office building floor $(27 \mathrm{~m} \times 41 \mathrm{~m})$ for eight configurations with different number of APs (from 5 to 39 , in which the 12 APs are numbered with \# in sub-figure (c).). The segments with different color represent different types of material (black: concrete, red: metal, grey: glass, yellow: wood, and green: layered drywall).

RSS, the standard deviation can be set to $\sigma_{P j}=3 \mathrm{~dB}[18]$, [19], $\forall j \in 1,2, \cdots, n$. According to [5], [7], the standard deviation of $\mathrm{ToF}$ is correlated with the distance between AP and mobile node, as well as the propagation in the line-of-sight (LoS) or non-line-of-sight (NLoS) situation. An empirical model of $\sigma_{\tau k}(k \in 1,2, \cdots, l)$ in nanosecond, is recommended by [5] as follows:

$$
\sigma_{\tau k}=\left\{\begin{array}{ll}
0.0614 d_{k}+0.3431, & \text { for } \operatorname{LoS} \\
0.1305 d_{k}+0.3063, & \text { for NLoS }
\end{array} .\right.
$$

Instead of CRLB, to compare the localization accuracy of different metrics, we define positioning error bound (PEB) as the square root of the lower bound $\sigma_{\mathrm{PEB}}(\mathbf{p})=\sqrt{\operatorname{tr}\left(\mathbf{I}^{-1}(\mathbf{p})\right)}$. The mean PEB $\bar{\sigma}_{\mathrm{PEB}}$ of all grid points (mobile nodes) are used to evaluate the localization performance.

\section{B. Comparison of Different Metrics}

To compare the positioning accuracy bound of the single metric scheme and hybrid metrics scheme, the numerical results of mean PEBs are shown in Fig. 3. In this simulation, we set the standard deviation of AoA $\sigma_{\alpha i}=7$ in degree for ease of comparisons, and the APs measure all AoA/RSS/ToF values. In Fig. 3, the single RSS metric obtains the worst positioning accuracy (the mean PEBs are always larger than one meter with varying number of APs), while the hybrid AoA/RSS/TOF localization achieves the best performance, which achieves the accuracy less than $0.51 \mathrm{~m}$ even though only 5 APs are deployed. Generally, the single metric of ToF, and the hybrid metrics incorporating $\mathrm{ToF}$ have excellent positioning performance, of which the mean PEBs are less than $0.65 \mathrm{~m}$. A minor accuracy improvement is achieved when incorporating RSS measurements in ToF-supporting APs, while adding AoA measurements can obtain a distinct increase of accuracy. Similarly, incorporating RSS metric to hybrid AoA/ToF-supporting APs, namely hybrid AoA/RSS/ToF, also gains quite small performance improvement.

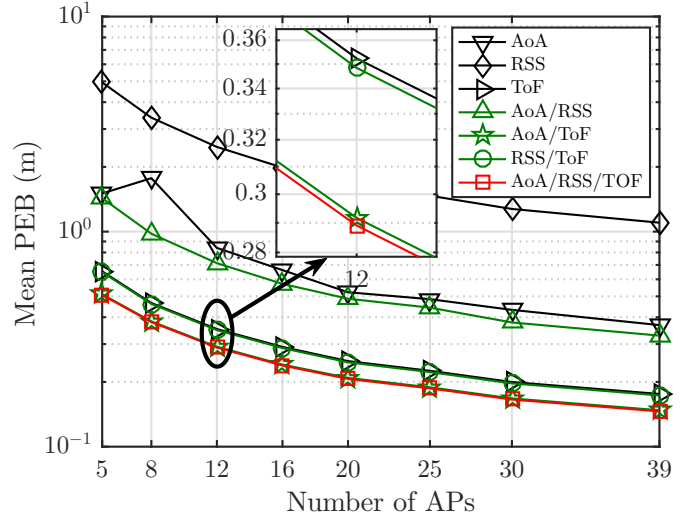

Fig. 3. Mean PEB of different metrics for varying number of APs with $\sigma_{\alpha i}=7$ in degree, $\sigma_{P i}=3 \mathrm{~dB}$, and $\sigma_{\tau i}$ given in (19).

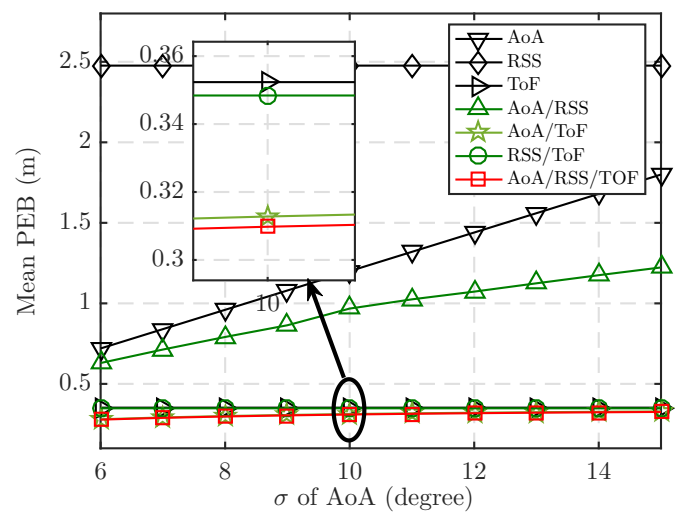

Fig. 4. Mean PEB of 12 APs of Fig. 2(c) with AoA's varying standard deviations.

In Fig. 4, we investigate the varying standard deviations of AoA's impact on the positioning accuracy. The number of APs is 12, as shown in Fig. 2(c), and the APs can 


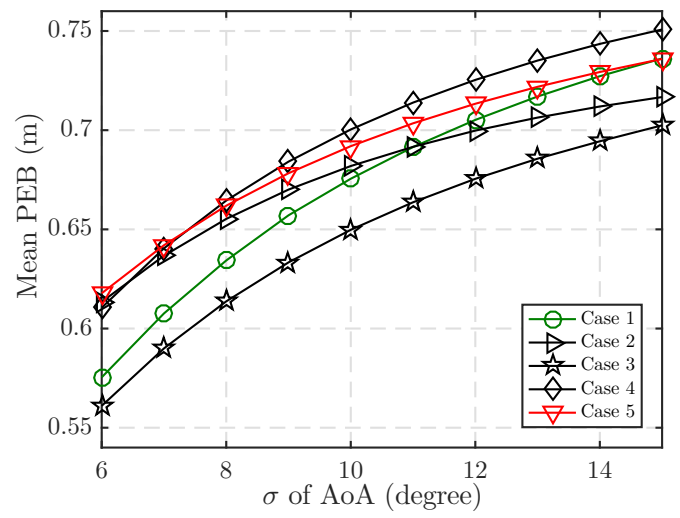

Fig. 5. Comparison of different deployments of hybrid AoA/RSS/ToF.

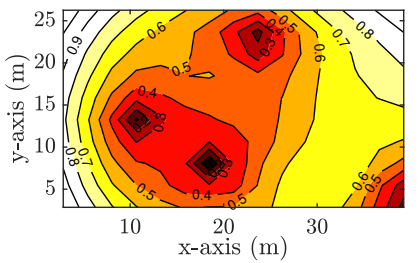

(a) Case 1

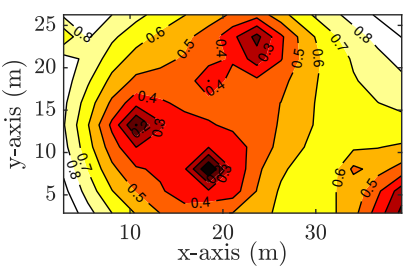

(c) Case 3

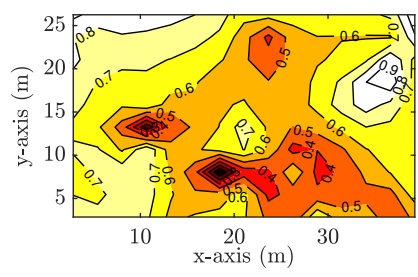

(e) Case 5

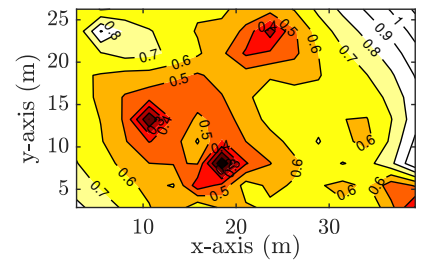

(b) Case 2

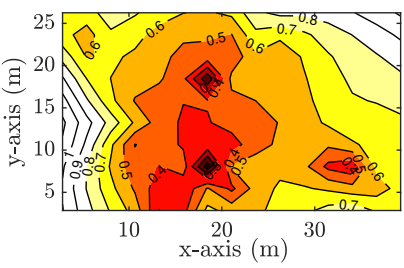

(d) Case 4

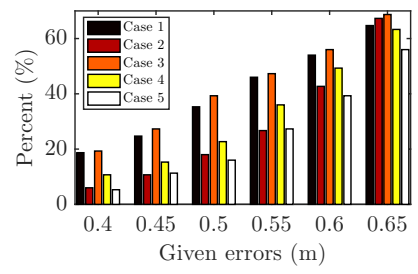

(f) Percentage
Fig. 6. The PEBs on the office building floor plane with $\sigma_{\alpha i}=7$ in degree, $\sigma_{P i}=3 \mathrm{~dB}$, and $\sigma_{\tau i}$ given in (19): (a)-(e) the distribution of five cases' PEBs, (f) the percentage of five cases when the PEBs are less than the given positioning errors.

measure all AoA/RSS/ToF values at the same time. We learn that the increasing of standard deviations deteriorates the localization performance of AoA-involved APs (including AoA metric, hybrid AoA/RSS metrics, hybrid AoA/ToF and hybrid AoA/RSS/ToF metrics). The mean PEB of AoA metric increases by $0.98 \mathrm{~m}$ from $0.72 \mathrm{~m}$ to $1.8 \mathrm{~m}$ when the standard deviation varies from 6 to 15 degrees, while the mean PEB for hybrid AoA/RSS metrics rises by $0.59 \mathrm{~m}$. However, the decrease is not obvious for hybrid $\mathrm{AoA} / \mathrm{ToF}$ and hybrid AoA/RSS/ToF metrics due to the good performance of ToFdominating positioning. Moreover, in line with Remark 3, the performance of hybrid metrics systems outperforms any of the corresponding single metric. Specifically, for AoAsupporting and AoA/RSS-supporting APs, a mean PEB of 1 $\mathrm{m}$ for AoA-supporting APs under standard deviation $\sigma_{\alpha i}=8^{\circ}$ can be obtained, while hybrid AoA/RSS metrics can reach the same accuracy when $\sigma_{\alpha i}$ is about 11 in degree. It reveals that hybrid metrics localization systems have better tolerance to large standard deviations.

\section{Influence of Different Hybrid AoA/RSS/ToF Schemes}

In practical applications, we cannot guarantee that each AP deployed supports all three metrics' measurements (AoA/RSS/ToF). Considering the cost, volume, and deployment of the APs, it is reasonable to expect that the APs only measure one of or two of the metrics. To compare the influence of different deployments of hybrid AoA/RSS/ToF metrics, we select the configuration in Fig. 2(c) with 12 numbered APs. Specially, five cases are defined below, all of which support four AoA measurements, four RSS measurements, and four ToF measurements. Three APs' subsets are introduced (Subset 1: $\# 1, \# 6, \# 8, \# 11$, Subset 2: \#2, \#3, \#4, \#9, Subset 3: $\# 5$, \#7, \#10, \#12). Notice that it cannot be expected to list all schemes of hybrid metrics, some typical cases allowing APs to be distributed as uniformly as possible, are given here.

- Case 1: 4 APs measure AoA/RSS/ToF with Subset 1.

- Case 2: 4 APs measure AoA/RSS with Subset 1, 4 APs measure ToF with Subset 2.

- Case 3: 4 APs measure AoA/ToF with Subset 1, 4 APs measure RSS with Subset 2.

- Case 4: 4 APs measure RSS/ToF with Subset 1, 4 APs measure AoA with Subset 2.

- Case 5: 4 APs measure AoA with Subset 1, 4 APs measure RSS with Subset 2, 4 APs measure AoA with Subset 3.

As shown in Fig. 5, the hybrid scheme of Case 3, under varying standard deviations of AoA, achieves the best positioning performance compared with other four hybrid schemes. Case 1 supporting all metrics' measurements simultaneously has relative high precision when the AoA's standard deviation $\sigma_{\alpha}$ is low (less than $10^{\circ}$ ), but the errors add distinctly as the $\sigma_{\alpha}$ increases. Fig. 6(a)-(e) presents the distributions of the PEBs under different hybrid schemes, and the percentage when the PEBs are less than given positioning errors. Generally, Case 3 achieves the best accuracy (sub-meter accuracy with $100 \%$, and 0.8-meter accuracy with 90\%) and largest coverage within an certain precision, which is further presented and interpreted by Fig. 6(f). Specifically, the coverage (percentage) of Case 3 with 0.5 -meter positioning errors is about $40 \%$ while the other cases only obtain the coverage less than $23 \%$ except for Case 1 with $35 \%$. As a result, it is recommended to adopt the hybrid scheme Case 3 and obtain a better positioning precision according to numerical results, which deserves subsequent experimental validation.

\section{CONCLUSION}

In this paper, the CRLB of hybrid AoA/RSS/ToF indoor positioning has been derived, considering the APs deployed 
may not support all three metrics. The existence conditions of the CRLBs of single/hybrid metrics, and the relationship between the CRLBs of single metric and of hybrid metrics, have also derived and revealed. Finally, numerical simulations based on iGent office floor plan data, show that hybrid metrics outperform single metric not only in positioning but also the robustness to standard deviations. Moreover, the hybrid scheme Case 3 is recommended for practical applications due to best accuracy and largest coverage under given accuracy compared with other schemes. A main future work will consist of experimental evaluation of hybrid metrics localization, especially the positioning performance of different hybrid AoA/RSS/ToF schemes in terms of system complexity and computation load.

\section{ACKNOWLEDGMENT}

This work is supported in part by the Excellence of Science (EOS) project MUlti-SErvice WIreless NETworks (MUSEWINET), and IMEC co-financed project InWareDrones.

\section{REFERENCES}

[1] J. Xiao, Z. Zhou, Y. Yi, and L. M. Ni, "A Survey on Wireless Indoor Localization from the Device Perspective," ACM Computing Surveys, vol. 49, no. 2, pp. 1-31, Jun 2016.

[2] M. Porretta, P. Nepa, G. Manara, and F. Giannetti, "Location, location, location," IEEE Vehicular Technology Magazine, vol. 3, no. 2, pp. 20 29, Jun 2008.

[3] T. J. Chowdhury, C. Elkin, V. Devabhaktuni, D. B. Rawat, and J. Oluoch, "Advances on localization techniques for wireless sensor networks: A survey," Computer Networks, vol. 110, pp. 284 - 305, Dec 2016.

[4] Q. D. Vo and P. De, "A survey of fingerprint-based outdoor localization," IEEE Communications Surveys Tutorials, vol. 18, no. 1, pp. 491-506, Firstquarter 2016.

[5] D. Plets, W. Deprez, J. Trogh, L. Martens, and W. Joseph, "Joint Received Signal Strength, Angle-of-Arrival, and Time-of-Flight Positioning," in 2019 13th European Conference on Antennas and Propagation (EuCAP), Apr 2019, pp. 1-5.

[6] I. Dotlic, A. Connell, H. Ma, J. Clancy, and M. McLaughlin, "Angle of arrival estimation using Decawave DW1000 integrated circuits," in 2017 14th Workshop on Positioning, Navigation and Communications (WPNC), Oct 2017, pp. 1-6.

[7] D. Vasisht, S. Kumar, and D. Katabi, "Sub-nanosecond time of flight on commercial Wi-Fi cards," in Proceedings of the 2015 ACM Conference on Special Interest Group on Data Communication, ser. SIGCOMM '15. New York, NY, USA: ACM, Aug 2015, pp. 121-122.

[8] S. Kay, Fundamentals of Statistical Signal Processing. Upper Saddle River, NJ: PTR Prentice Hall, 1993.

[9] J. Xu, M. Ma, and C. L. Law, "AoA cooperative position localization," in IEEE GLOBECOM 2008 - 2008 IEEE Global Telecommunications Conference, Nov 2008, pp. 1-5.

[10] A. K. M. M. Hossain and W. Soh, "Cramer-Rao bound analysis of localization using signal strength difference as location fingerprint," in 2010 Proceedings IEEE INFOCOM, Mar 2010, pp. 1-9.

[11] J. Shen, A. F. Molisch, and J. Salmi, "Accurate passive location estimation using ToA measurements," IEEE Transactions on Wireless Communications, vol. 11, no. 6, pp. 2182-2192, Jun 2012.

[12] A. Catovic and Z. Sahinoglu, "The Cramer-Rao bounds of hybrid TOA/RSS and TDOA/RSS location estimation schemes," IEEE Communications Letters, vol. 8, no. 10, pp. 626-628, Oct 2004.

[13] F. Shang, B. Champagne, and I. N. Psaromiligkos, "A ML-based framework for joint ToA/AoA estimation of UWB pulses in dense multipath environments," IEEE Transactions on Wireless Communications, vol. 13, no. 10, pp. 5305-5318, Oct 2014.

[14] Y. Li, G. Qi, and A. Sheng, "Performance metric on the best achievable accuracy for hybrid TOA/AOA target localization," IEEE Communications Letters, vol. 22, no. 7, pp. 1474-1477, Jul 2018.
[15] D. Plets, W. Joseph, K. Vanhecke, E. Tanghe, and L. Martens, "Simple indoor path loss prediction algorithm and validation in living lab setting," Wireless Personal Communications, vol. 68, no. 3, pp. 535-552, Feb 2013.

[16] WAVES UGent-imec, "WiCa Heuristic Indoor Propagation Prediction Tool (WHIPP, expert edition)," [Online]. Available: https://www.waves.intec.ugent.be/exposure-tool/expert-edition.

[17] M. Kotaru, K. Joshi, D. Bharadia, and S. Katti, "SpotFi: Decimeter level localization using WiFi," in 2015 ACM Conference on Special Interest Group on Data Communication (Sigcomm '15), Aug 2015, pp. 269-282.

[18] V. Erceg et al., "TGn channel models," May 2004, IEEE P802.11, 802.11-03/940r4.

[19] R. Porat, S. Yong, and K. Doppler, "TGah channel model," Mar 2015, IEEE P802.11, 802.11-11/0968r4. 